\title{
Revista Brasileira de Enfermagem REBEn \\ Aplicação da Técnica de Estrutura Analítica de Projeto para o sub-projeto do Catálogo de Sites da Biblioteca Virtual em Saúde - Enfermagem
}

\author{
Application of the Technique of Analytical Structure of Project for the sub-project of \\ Websites Catalog of the Virtual Health Library - Nursing \\ Aplicación de la Técnica de Estructura Analítica de Proyecto para el subproyecto de lo \\ Catalogo de Sitios de la Biblioteca Virtual en Salud - Enfermería
}

\section{Luís Augusto dos Santos}

Engenheiro Eletricista. Aluno do Programa de Mestrado do Departamento de Enfermagem da Universidade Federal de São Paulo (UNIFESP), São Paulo, SP. Membro do Núcleo de Informática em Enfermagem (NIEn).

\section{Heimar de Fátima Marin}

Enfermeira. Professora Titular do Departamento de Enfermagem da UNIIFESP, São Paulo, SP. Líder do NIEn.

Isaac Rosa Marques

Enfermeiro. Aluno do Programa de

Doutorado do Departamento de Enfermagem da UNIFESP, São Paulo, SP. Secretário do NIEn.

Isabel Cristina Kowal OIm Cunha

Enfermeria. Doutora em Saúde Pública. Professora Adjunto do Departamento de Enfermagem da UNIFESP, São Paulo, SP. Membro do NIEn.

Sub-projeto realizado pelo Núcleo de Informática em Enfermagem (NIEn) para o Projeto BVS - Enfermagem.

\section{RESUMO}

Este trabalho procura de forma didática, explicar os benefícios de uso de uma técnica de gerenciamento de projetos, chamada de Estrutura Analítica do Projeto - uma ferramenta gráfica para identificar os resultados principais a serem desenvolvidos por um projeto. Os exemplos são reais, aplicados a um sub-projeto da Biblioteca Virtual em Saúde em Enfermagem (BVS-Enfermagem) para o desenvolvimento de Catálogo de Sites. Destacam-se os benefícios de visualização gráfica para um maior entendimento entre profissionais de diferentes áreas.

Palavras-chave: Projeto de sistemas; Formulação de projetos; Informática em enfermagem.

\section{ABSTRACT}

This work search of didactic form to explain the benefits of use of one technique of management project, called Work Breakdown Structure: a graphical tool to identify the main results to be developed for a project. The real examples are applied to a sub-project of the Virtual Library in Health in Nursing (BVSEnfermagem) to development of the Sites Catalogs. It is distinguished the benefits of graphical visualization for a major agreement between professionals of different expertise. Descriptors: System design; Programming; Nursing informatics.

\section{RESUMEN}

Este artículo presenta de una forma didáctica algunas explicaciones sobre los beneficios de lo uso de la técnica de gestión de proyectos, llamada de Estructura Analítica del Proyecto - una herramienta grafica para la identificación de los principales resultados que han de ser desarrollados por un proyecto. Los ejemplos son reales, aplicados a un subproyecto de la Biblioteca Virtual en Salud - Enfermería (BVSEnfermagem) para el desarrollo del catalogo de sitios. Son destacados los beneficios de visualización grafica para un mejor entendimiento entre profesionales de diferentes áreas.

Descriptores: Diseño de sistemas; Programación; Informática en enfermería.

Santos LA, Marin HF, Marques IR, Cunha ICKO. Aplicação da Técnica de Estrutura Analítica de Projeto para o sub-projeto do Catálogo de Sites da Biblioteca Virtual em Saúde - Enfermagem. Rev Bras Enferm 2007 nov-dez; 60(6): 716-20.

\section{INTRODUÇÃO}

Os projetos são esforços temporários desenvolvidos de forma progressiva, porém não são operações contínuas. São empreendidos para criar um produto, serviço ou um resultado único. É de caráter temporário, pois tem um início e um fim definidos, isto é, esse fim é quando os objetivos do projeto são conquistados. A elaboração progressiva é uma característica de projetos que integra os conceitos de temporário e único(1).

É recomendável que um projeto inicie formalmente através de um documento, um Termo de Abertura de projeto - TA, contendo nome ou sigla, necessidade, justificativa, objetivo, requisitos principais, escopo preliminar sucinto, quais são as premissas e as restrições, estimativas de prazo e custo; riscos prováveis e um aceite do patrocinador e ou cliente do projeto. É interessante declarar no documento o que não inclui nesse escopo, como exemplo desenvolver um sistema e que não haverá compra de equipamentos especializados.

A necessidade é descrita através de um problema a ser resolvido ou uma oportunidade a ser alcançada ou ainda uma exigência legal a ser cumprida. A justificativa é o que irá agregar de valor a organização ao solucionar a necessidade, ou seja, o porquê da existência do projeto. O objetivo deve ser descrito de forma específica, mensurável, acordado, realístico e definido no tempo. Os requisitos são uma relação de "0 quê"
Submissão: 20/07/2007

Aprovação: 21/10/2007 
é necessário desenvolver. O escopo preliminar é "o como" fazer os requisitos, ressaltando que suas exclusões também devem ser declaradas, pois pode haver a ocorrência de aumento do escopo, alterando o prazo e custo. Premissas são os fatos aceitos como verdadeiros no início do projeto e restrições são limitadores de opções do projeto. Estimativas são concebidas como ordem de grandeza de prazo e custo do projeto. Riscos são eventos futuros e incertos que podem atingir o prazo, o custo e o escopo.

Uma vez aprovado o projeto, através do documento de TA, inicia-se 0 documento Declaração Preliminar do Escopo do projeto - DPE, aqui não utilizado para efeitos didáticos, apenas o item do escopo preliminar sucinto. 0 escopo, ou seja, o "como" vamos atender os requisitos, é a fonte de informação da Estrutura Analítica do Projeto - EAP, que representa as subdivisões do projeto de forma gráfica, suas principais entregas em componentes menores e mais facilmente gerenciáveis.

\section{CONCEITO DA ESTRUTURA ANALÍTICA DO PROJETO - EAP}

A EAP é uma decomposição hierárquica orientada à entrega do trabalho. Não é uma definição de atividades, o foco deve ser nas entregas (deliverables). Uma entrega é qualquer produto, resultado ou capacidade para realizar um serviço(2), aqui será referenciado como produtos (ou serviços e documentos necessários) a serem executados pela equipe do projeto para atingir os objetivos do mesmo e criar as entregas necessárias. Nesta fase, organizase e define o escopo total do projeto; subdivide o trabalho do projeto em partes menores e mais facilmente gerenciáveis, quais sejam, os componentes, em que cada nível descendente da EAP representa uma definição cada vez mais detalhada do trabalho do projeto.

Os componentes que formam a EAP auxiliam as partes interessadas em visualizar e comunicar as entregas do projeto. Ao definir o produto pode-se monitorar e controlar o trabalho planejado contido nos componentes de nível mais baixo da EAP, denominados pacotes de trabalho (work packages). Estes sim é que deverão ser decompostos em atividades.

A EAP auxilia também a retornar, ou melhor, a redefinir o escopo, ou seja, a estabelecer quais são as atividades que, quando ordenadas, podem ser agendadas. Inclui aqui também a determinação de quais recursos materiais e humanos serão necessários, portanto, a estimativa de custos e quais são as áreas críticas de qualidade e os riscos envolvidos.

Em resumo, é a mais importante ferramenta de planejamento, é o insumo fundamental para a resposta de todas as perguntas que temos de responder para o planejamento, facilita a definição de responsabilidades e estabelece a base de referência para controle.

\subsection{A técnica EAP}

Ao construir uma EAP deve-se ter em mente o produto a ser entregue, 0 que é requerido e necessário a ser providenciado, quais são os componentes que contribuem para caracterizar o final do projeto. Um produto de um projeto é qualquer resultado ou item, mensurável, tangível ou um efeito verificável, que deve ser originado como parte do projeto. O requerido e necessário é "o que" será entregue ao longo da vida do projeto. Os componentes caracterizam subdivisões dos produtos principais a serem entregues.

Podem-se definir quais métodos, processos, requisitos que também devem ser seguidos, assim como um conjunto de, áreas de atenção de qualidade, de inspeções e de riscos potenciais.

É recomendável que siga os quatro maiores passos da decomposição, quais sejam: (a) identificar o produto final do projeto; (b) definir os maiores ou os principais produtos; (c) decompor os maiores produtos em um nível de detalhe para gerenciamento e controle; (d) rever e refinar a EAP até atingir uma concordância dos envolvidos.

Cada componente daEAP representa apenas um produto com a integração de todos abaixo desse, é único, distinto dos demais e deve ser claramente definido, também há apenas um único "componente-pai". A EAP deve ser vista como uma lista de marcos importantes. É usual que a maioria dos pacotes de trabalho sejam orçados entre $0,5 \%$ a $2,5 \%$ do orçamento total. Ainda, não é necessário que todos os ramos tenham o mesmo número de níveis e sempre deve-se definir uma equipe ou uma pessoa como responsável pelo produto.

A figura 1, a seguir, exibe um projeto exemplo que contem fases e subprojetos é apresentado.

Não há atividades descritas. Não há tempos e custos, uma vez que os mesmos são baseados nas atividades. O propósito é estabelecer uma comunicação gráfica entre o escopo do trabalho e os envolvidos no projeto para ser criticado e aprimorado progressivamente o trabalho do projeto. As

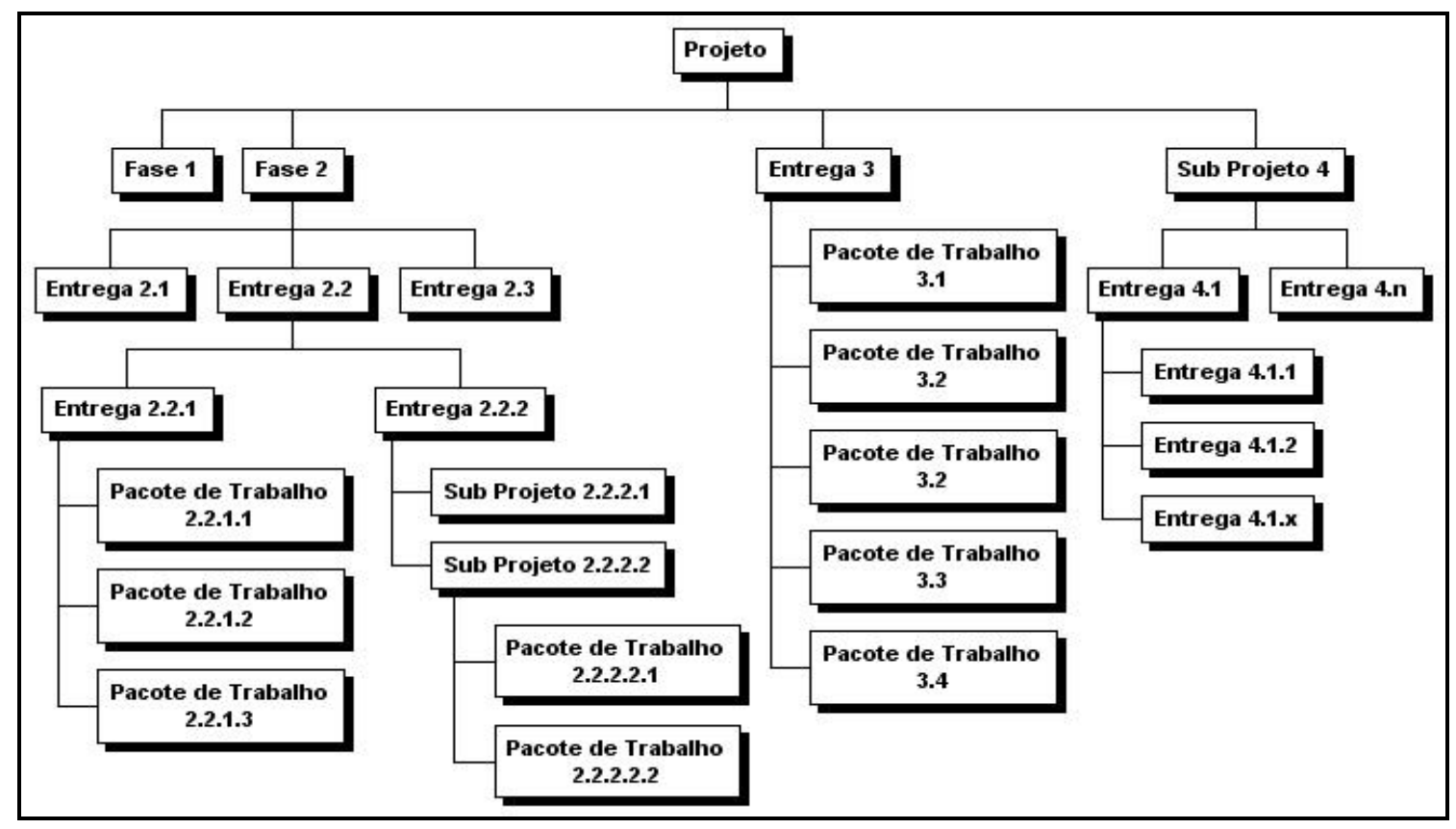

Figura 1. Exemplo de uma EAP. 
atividades serão somente descritas após a concordância dos envolvidos, ou seja, se o que está representado de forma gráfica é o trabalho a ser entregue.

A figura 1, tem a intenção de comunicar que o projeto possui em seu nível mais alto, chamado de "Nível 1", respectivamente, duas fases, uma entrega e um sub-projeto, ("Fase 1", "Fase 2", "Entrega 3" e "Sub Projeto 4".

Por exemplo, a "Fase 1" representa um levantamento inicial de necessidades, a "Fase 2" um desenvolvimento de um sistema, com seus produtos ou serviços, representados em um segundo nível, as "Entregas 2.1; 2.2 e 2.3", respectivamente, uma definição de requisitos, um desenvolvimento de um aplicativo de software e por último um treinamento. A representação de um terceiro nível, a "Entrega 2.2", o aplicativo, que estaria dividida em dois produtos, a "Entrega 2.2.1" as funcionalidades requeridas e a "Entrega 2.2.2" uma interface com outros sistemas.

A "Entrega 3" poderia representar uma obra civil, parte do projeto, como os membros da equipe não possuem essa perícia devem repassar a outros envolvidos no projeto. Por fim o "Sub Projeto 4" uma nova área ou um conjunto de produtos. Esses dois produtos estão também representados em mais níveis decompostos.

Essas explicações são chamadas de "Dicionário da EAP" e constituem parte fundamental para os registros da organização do trabalho do projeto.

\subsection{O Projeto BVS - Enfermagem}

O Projeto BVS - Enfermagem é compreendido por 13 sub-projetos, um desses é o "Catálogo de Sites em Enfermagem", aqui descrito e sob a responsabilidade do Departamento de Enfermagem da UNIFESP, através de seu Núcleo de Informática em Enfermagem (NIEn).

O Centro Latino-Americano e do Caribe de Informação em Ciências da Saúde - BIREME desenvolveu, com êxito, a partir de 1967, a capacidade dos países da Região de criar e operar sistemas nacionais de informação técnico-científicas, em sintonia com as novas tecnologias de informação e comunicação, bem como com os novos paradigmas organizacionais e de tratamento da informação. Nos últimos anos, a demanda de cooperação técnica de produtos e serviços baseados na Internet exigiu mudanças significativas ${ }^{(3)}$.

Entendo o projeto BIREME como um grande sucesso, a BVS Enfermagem permite a construção de patrimônio informacional em enfermagem, com fácil acesso, sem preocupações com tempo, espaço e fronteiras, viabilizando melhorar qualidade da educação, impulsionando os processos da geração de conhecimento, melhorando a formação de recursos humanos de enfermagem uma vez que o desenvolvimento científico e tecnológico é necessário para alcançar o objetivo final de elevar a qualidade de vida da população no Brasil e na América Latina e Caribe ${ }^{(4)}$.

E uma de suas justificativas é expandir para outros países da América
Latina e Caribe a metodologia e os componentes da BVS - Enfermagem, através de uma base de dados única de enfermagem com apoio dos centros nacionais de cooperação técnica, coletando dados da literatura produzida em cada país, exercendo assim o controle bibliográfico nacional da área.

\subsection{Termo de Abertura}

Conforme já mencionado, um TA de projeto deve conter, as necessidades, as justificativas, um objetivo, as premissas, as restrições, um escopo sucinto e preliminar e suas exclusões, um prazo e custo estimados.

É um documento principal e inicial para registrar as necessidades de negócios e de novo produto, serviço ou outro resultado que deve satisfazer estes requisitos ${ }^{(1)}$, como o escopo pretendido, o prazo acordado, o custo orçado e a qualidade exigida.

A seguir um formato de documento proposto para registrar o projeto, este é o documento inicial onde é descrito de forma resumida as principais informações do projeto (veja a tabela 1).

OTAé uma redação técnica cada parágrafo é uma ligação do anterior, um documento inicial de projeto para orientar as decisões futuras, onde se declara a qualidade requerida nos itens de necessidade, justificativa e objetivo. Também os riscos em premissas e restrições devem ser incluídos.

\subsection{A EAP do Catálogo de Sites da BVS-Enfermagem}

A EAP é baseada no escopo, mais precisamente em um documento de Declaração Preliminar de Escopo, aqui utilizado através do documento de TA em seu item escopo preliminar vide figura 2.

A leitura do escopo preliminar fornece as orientações básicas para a EAP, também as reuniões com os patrocinadores do projeto e documentos de apoio ou de detalhes fornecidos são fontes para complementar a construção da EAP.

A figura 2 está representado uma boa prática que é o produto "Gerenciamento de Projeto" e seus sub-produtos. O "sign-on" ou autorização para o início do projeto, normalmente declarado como a data no TA. O "Planejamento do Projeto" que é composto por outras atividades de planejamento como elaboração de cronograma, os cálculo de custos, a definição de qualidade requerida, os recursos humanos a serem atribuídos, contratados ou treinados, o plano de comunicação relatando o progresso do projeto, o plano de tratamento de riscos e plano de aquisições, todos referenciados no guia PMBOK®(1). O "Acompanhamento do Projeto" que visa a verificar, corrigir ou alterar os planos do projeto. Por fim o "sign-off" ou aceite final do projeto, caracterizando formalmente o encerramento do projeto.

Cada componente representado na EAP deve vir acompanhando de uma descrição, chamada de "Dicionário da EAP". Uma forma resumida dos componentes restantes está descrita na Tabela 2.

\section{Tabela 1. TA do Catálogo de Sites de Enfermagem}

\begin{tabular}{|c|c|}
\hline Termo de Abertura & Data: 02/11/2005 \\
\hline Projeto: Catálogo de Sites de Enfermagem & Patrocinador: BIREME \\
\hline Gerente: LA & Cliente: Rede BVS - enfermagem \\
\hline Equipe Básica: AF & Equipe de Suporte: IN \\
\hline \multicolumn{2}{|c|}{$\begin{array}{l}\text { Necessidades do projeto (o problema a ser solucionado): "Há uma dificuldade cada vez mais freqüente de identificação de fontes de informação fidedignas em } \\
\text { meio a uma quantidade enorme de informação recuperada em uma busca efetuada sobre algum tema em saúde." }\end{array}$} \\
\hline \multicolumn{2}{|c|}{$\begin{array}{l}\text { Justificativas (o valor a ser gerado pelo projeto): "Contribuir para o acesso universal às fontes de informação em saúde mediante o desenvolvimento do } \\
\text { catálogo de fontes de informação disponíveis na Internet para a promoção e uso intensivo de fontes de informação científico-técnica em saúde." }\end{array}$} \\
\hline \multicolumn{2}{|c|}{ Objetivo: Desenvolver o catálogo de sites de informação em Enfermagem disponíveis na Internet, até 30/06/2006. } \\
\hline \multicolumn{2}{|c|}{$\begin{array}{l}\text { Premissas (fatos aceitos): "Uso da metodologia de registro e recuperação de fontes do Localizador de Informação em Saúde - LIS, um dos componentes } \\
\text { integradores da BVS. As fontes de informação em enfermagem e áreas afins disponíveis na Internet em âmbito nacional, regional e internacional e será } \\
\text { desenvolvido e operado na Biblioteca Virtual em Saúde." }\end{array}$} \\
\hline \multicolumn{2}{|c|}{$\begin{array}{l}\text { Restrições (fatores que limitam o projeto): "Dificuldade cada vez mais freqüente de identificação de fontes de informação fidedignas em meio a uma quantidade } \\
\text { enorme de informação recuperada em uma busca efetuada sobre algum tema em saúde. Finalizar em 30/06/2006" }\end{array}$} \\
\hline \multicolumn{2}{|c|}{$\begin{array}{l}\text { Escopo preliminar (como será feito o projeto): "Um catálogo exaustivo de fontes de informação em enfermagem disponíveis na Internet; Um mecanismo de } \\
\text { atualização sistematizada das fontes de informação em enfermagem disponíveis na Internet; Desenvolver e operar o catálogo na BVS;" }\end{array}$} \\
\hline \multicolumn{2}{|c|}{ Exclusões do escopo: "Fonte de informação não certificada." } \\
\hline & \\
\hline
\end{tabular}




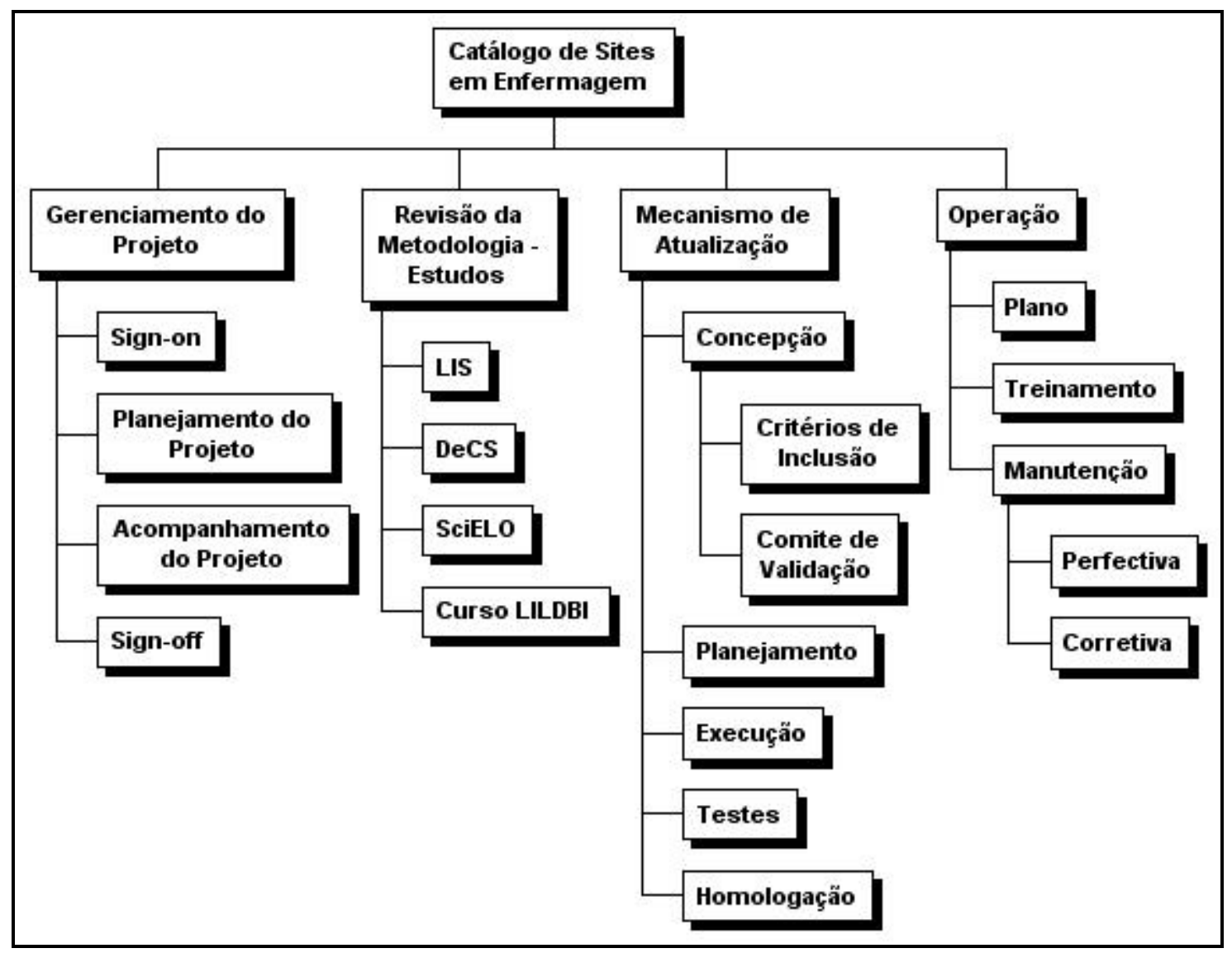

Figura 2. EAP do Catálogo de Sites da BVS - Enfermagem.

Tabela 2. Dicionário da EAP.

\begin{tabular}{|c|c|}
\hline Dicionário - EAP & Data: 02/11/05 \\
\hline Projeto: Catálogo de Sites em Enfermagem & Patrocinador: $\mathrm{BOO}$ \\
\hline \multicolumn{2}{|c|}{ Gerenciamento do Projeto: A autorização para inicio (sign-on), o planejamento do projeto, o acompanhamento e a declaração de encerramento (sign-off). } \\
\hline \multicolumn{2}{|c|}{$\begin{array}{l}\text { Revisão da Metodologia: a ser utilizada, o estudo do Localizador de Informações em Saúde - LIS, o estudo da terminologia Descritores em Ciências da Saúde } \\
\text { - DeCS, o estudo da metodologia Scientific Electronic Library Online - SciELO e a equipe que irá fazer o curso LILDBI sobre a base de dados Literatura Latino } \\
\text { Americana e do Caribe de Informação em Ciências de Saúde - LILACS. }\end{array}$} \\
\hline \multicolumn{2}{|c|}{$\begin{array}{l}\text { Mecanismo de Atualização: dos sites, concepção dos Critérios de seleção para o cadastro de fontes de informação em enfermagem disponíveis na Internet, e } \\
\text { definição do Comitê de Validação um núcleo de instituições responsáveis pelo registro das fontes de informação técnico-científica em enfermagem } \\
\text { participantes da Sub-Rede de Informação em Enfermagem - SURENF. O planejamento, a execução, os testes e a homologação devem ser definidos junto a } \\
\text { equipe de projeto. }\end{array}$} \\
\hline \multicolumn{2}{|c|}{$\begin{array}{l}\text { Operação: com os seus componentes de planos de Treinamento para atualização regular do conteúdo do novo produto Catálogo de Sites de Enfermagem, e o } \\
\text { trabalho das manutenções perfectiva e corretiva através de implantação de níveis de responsabilidade para a atualização e integridade das fontes de } \\
\text { informação entre as instituições participantes. }\end{array}$} \\
\hline
\end{tabular}

O importante a destacar é que aEAP procura estabelecer a comunicação gráfica de diversos documentos iniciais do projeto identificando os produtos a serem entregues durante eao final do projeto, que uma vez feitos os acordos entre os envolvidos o ponto de partida para o desenvolvimento de planos de projeto.

\section{CONCLUSÃO}

A técnica EAP demonstra ser um grande auxílio entre profissionais de diferentes áreas para comunicar o trabalho a ser empreendido, quer através de um projeto ou para um comum entendimento de resultados que uma organização produz através de produtos e ou serviços.

Ė umas das técnicas principais utilizadas por gerentes de projetos para estabelecer uma comunicação efetiva aos envolvidos no projeto. Também possibilita identificar os pontos de maior qualidade requerida e os riscos potenciais a serem enfrentados e possivelmente evitados.

Todo o desenho da EAP, uma vez entendido e acordado entre os envolvidos, deverá então seguir para a próxima etapa, que irá definir as atividades para cada pacote de trabalho, onde estarão concentradas nestes componentes, necessitando o comprometimento da equipe com os prazos a serem definidos de comum acordo.

AEAP é uma ferramenta anterior a uma definição de atividades, cronograma e custo de maior precisão, seu foco primário é em "o que" vai ser feito, caracterizando também uma progressiva comunicação entre as diferentes áreas envolvidas em um projeto. 
Santos LA, et al.

\section{REFERÊNCIAS}

1. Project Management Institute. Practice standard for work breakdown structures. Newtown Square (PA): Project Managment Institute; 2001.

2. Project Management Institute. A guide to the project management body of knowledge $3^{\text {rd }}$ ed. Newtown Square (PA): Project Management Institute; 2004.
3. Lana FCF, Ribeiro MPF, Piazza M, Abdala V, organizadores. BVS - Enfermagem: projeto para desenvolvimento e operacionalização. Belo Horizonte (MG): Ministério da Saúdel BIREME/OPAS/OMS; 2005.

4. BIREME/OPAS/OMS. Biblioteca Virtual em Saúde - Enfermagem. São Paulo (SP); 2006. [citado em: 2007 jul 10]. Disponível em: URL: http://enfermagem.bvs.br/html/pt/home.html 